\title{
THE BÉZIER VARIANT OF LUPAS KANTOROVICH OPERATORS BASED ON POLYA DISTRIBUTION
}

\author{
Bo-Yong LIAN AND QING-Bo CAI
}

\begin{abstract}
In this paper we introduce the Bézier variant of Lupas Kantorovich operators based on Polya distribution. We establish a direct approximation by means of the Ditzian-Totik modulus of smoothness and a global approximation theorem in terms of second order modulus of continuity. Furthermore, we give the rate of convergence for absolutely continuous functions having a derivative equivalent to a bounded function. Our results extend the work of Agrawal [P. N. Agrawal, N. Ispir and A. Kajla, Approximation properties of Lupas-Kantorovich operators based on polya distribution, Rendiconti del Circolo Matematico di Palermo Series 2, 2016, 65 (2): 185-208] and Ispir [N. Ispir, P. N. Agrawal and A. Kajla, Rate of convergence of Lupas Kantorovich operators based on Polya distribution, Appl. Math. Comput., 2015, 261: 323-329].
\end{abstract}

Mathematics subject classification (2010): 41A36, 41A25, 41A10.

Keywords and phrases: Bézier operators, Polya distribution, rate of convergence, absolutely continuous functions.

\section{REFERENCES}

[1] L. Lupas AND A. LuPas, Polynomials of binomial type and approximation operators, Stud. Univ. Babes-Bolyai Math., 1987, 32 (4): 61-69.

[2] D. Miclaus, The revision of some results for Bernstein-Stancu type operators, Carpathian J. Math., 2012, 28 (2): 289-300.

[3] V. GuPTA AND T. M. Rassias, Lupas-Durrmeyer operators based on polya distribution, Banach J. Math. Anal., 2014, 8 (2): 146-155.

[4] P. N. Agrawal, N. Ispir And A. KaJla, Approximation properties of Lupas-Kantorovich operators based on polya distribution, Rendiconti del Circolo Matematico di Palermo Series 2, 2016, 65 (2): $185-208$.

[5] N. Ispir, P. N. Agrawal And A. KaJla, Rate of convergence of Lupas Kantorovich operators based on Polya distribution, Appl. Math. Comput., 2015, 261: 323-329.

[6] A. Aral and V. Gupta, Direct estimates for Lupas-Durrmeyer operators, Filomat, 2016, 30 (1): 191-199.

[7] V. Gupta, A. M. Acu And D. F. Sofonea, Approximation of Baskakov type Polya-Durrmeyer operators, Appl. Math. Comput., 2017, 294: 318-331.

[8] V. Gupta And D. SoyBas, Convergence of integral operator based on different distributions, Filomat, 2016, 30 (8): 2277-2287.

[9] V. Gupta AND R. P. Agarwal, Convergence Estimates in Approximation Theory, Springer International Publishing, Switzerland, 2014.

[10] X. M. Zeng AND A. PIRIou, On the rate of convergence of two Bernstein-Bézier type operators for bounded variation functions, J. Approx. Theory, 1998, 95 (3): 369-387.

[11] X. M. ZENG, On the rate of convergence of two Bernstein-Bézier type operators for bounded variation functions II, J. Approx. Theory, 2000, 104 (2): 330-344.

[12] V. Gupta AND X. M. Zeng, Rate of approximation for the Bézier variant of Balazs Kantorovich operators, Math. Slovaca, 2007, 57 (4): 349-358.

[13] B. Y. Lian, Rate of approximation of bounded variation functions by the Bézier variant of Chlodowsky operators, J. Math. Inequal., 2013, 7 (4): 647-657. 
[14] X. M. ZENG AND B. Y. LiAn, An estimate on the convergence of MKZ-Bézier operators, Comput. Math. Appl., 2008, 56 (12): 3023-3028.

[15] P. N. Agrawal, N. IspiR AND A. KaJla, Approximation properties of Bézier-summation-integral type operators based on Polya-Bernstein functions, Appl. Math. Comput., 2015, 259: 533-539.

[16] T. NEer, A. M. ACU AND P. N. AgRAwAL, Bézier variant of genuine-Durrmeyer type operators based on Polya distribution, Carpathian J. Math., 2016, 33 (1): 73-86.

[17] R. A. Devore And G. G. Lorentz, Construtive Approximation, Springer-Verlag, Berlin, 1993.

[18] Z. Ditzian And V. Totik, Moduli of Smoothness, Springer, New York, 1987.

[19] R. BOJANIC AND F. CHENG, Rate of convergence of Bernstein polynomials for functions with derivatives of bounded variation, J. Math. Anal. Appl. 1989, 141 (1): 136-151. 